\title{
Concentration dependence of terahertz generation in jets of water and ethanol mixtures
}

\author{
A. N. Tcypkin, E. A Ponomareva, S. E. Putilin, S. V. \\ Smirnov, S. A. Shtumpf, et al.
}

A. N. Tcypkin, E. A Ponomareva, S. E. Putilin, S. V. Smirnov, S. A. Shtumpf, M. V. Melnik, Y. E, S. A. Kozlov, X.-C. Zhang, "Concentration dependence of terahertz generation in jets of water and ethanol mixtures," Proc. SPIE 10826, Infrared, Millimeter-Wave, and Terahertz Technologies V, 1082603 (9

November 2018); doi: 10.1117/12.2501217

SPIE. Event: SPIE/COS Photonics Asia, 2018, Beijing, China 


\title{
Concentration dependence of terahertz generation in jets of water and ethanol mixtures
}

\author{
A.N. Tcypkin, ${ }^{1,} *$ E.A. Ponomareva, ${ }^{1}$ S.E. Putilin, ${ }^{1}$ S.V. Smirnov, ${ }^{1}$ S.A. Shtumpf, ${ }^{1}$ \\ M.V. Melnik, ${ }^{1}$ Y. E, ${ }^{2}$ S.A. Kozlov ${ }^{1}$ and X.-C. Zhang ${ }^{1,2,3}$ \\ ${ }^{1}$ ITMO University, Saint-Petersburg 197101, Russia \\ ${ }^{2}$ The Institute of Optics, University of Rochester, Rochester, NY 14627, USA \\ ${ }^{3}$ Beijing Advanced Innovation Center for Imaging Technology, Capital Normal University, \\ Beijing 100037, China
}

*Corresponding Author: tsypkinan@gmail.com

In this work, we considered mixtures of ethanol and water in the form of jets as samples for $\mathrm{THz}$ generation based on laser-induced filamentation. The dependence of the output energy of terahertz radiation on the concentration of ethanol in water was experimentally studied. It is shown that the energy grows linearly, which can be explained by an increase in the ionization energy due to the linear replacement of low-efficient charge carriers (water) with highlyefficient (ethanol). The dependence of the $\mathrm{THz}$ generation on the optical angle of incidence on the mixture jets was also demonstrated. The results of this study can be further used to create universal source of terahertz radiation.

Key words: terahertz generation, ethanol, ultrashort pulses, plasma 
$\mathrm{I}_{\mathrm{n}}$ n current terahertz spectroscopy the sources of $\mathrm{THz}$ radiation based on laser-induced filamentation are becoming increasingly important. The issue of obtaining radiation in this range is relevant since broadband terahertz radiation is suitable for a wide range of applications, for example, in nonlinear terahertz optics [1].

The main parameter of the $\mathrm{THz}$ radiation sources is efficiency. There are highly efficient methods of $\mathrm{THz}$ generation, for example, from crystals, which are limited by the destruction of the material with a further increase in the pump energy [2]. The most popular mechanism is terahertz generation via two-color filamentation in gases due to the possibility to control output radiation by the input laser parameters [3]. However, the search for new sources is unlimited and opens a vast area for further investigation.

Although the use of liquids as $\mathrm{THz}$ sources certainly has a great potential, it has not yet been studied to the full extent. At the moment, there are two known approaches on the generation of terahertz radiation in a water film and a cell $[4,5]$. However, the use of film led to the destruction of the liquid samples at energies above $500 \mu \mathrm{J}$. In the case of cell, there are restrictions on its material. Using the jet, we avoid these drawbacks and obtain the possibility of increasing the pump energy.

Here, we demonstrate optimal conditions of terahertz generation in liquid jets, based on experimental investigations of ethanol and water mixtures. We study the dependence of the terahertz energy on the ethanol concentration. Considering that the generation of radiation through the plasma channel is determined by the number of current carriers at the external atomic level, it was expected that the growth of ethanol molecules would lead to an increase in the generation efficiency due to the higher ionization energy of ethanol. We experimentally showed that the increase in energy is linear. Here we also demonstrate that changing the concentration of ethanol in the mixture is lead to a shift in the optimal angle of incidence of radiation on the jet. This phenomenon has already been studied and explained for water film in [6]. Thus, the obtained results should be considered when studying the conditions of terahertz generation in liquids.

\section{Results}

Experimental setup. The excitation laser has the central wavelength of $800 \mathrm{~nm}$, the pulse energy from 0.01 to $2 \mathrm{~mJ}$, the repetition rate of $1 \mathrm{kHz}$, and the pulse duration varied from $35 \mathrm{fs}$ to $700 \mathrm{fs}$. The pump radiation was focused with a $5 \mathrm{~cm}$ focal length parabolic mirror on the water liquid jet $(140 \mu \mathrm{m}$ and $300 \mu \mathrm{m})$ where the terahertz radiation was generated. The jet was installed on the translator, which allowed it to rotate, changing the radiation angle of incidence $\alpha$ on the jet. To register terahertz radiation, we used a standard scheme of a terahertz spectrometer based on the electro-optical effect. Here we used ZnTe crystal $1 \mathrm{~mm}$ thick, which made it possible to detect a signal up to $3 \mathrm{THz}$.

An illustration of incident angle $\alpha$ is shown in the inset of Fig. 1, where $\hat{n}$ is the surface normal of the jet. 


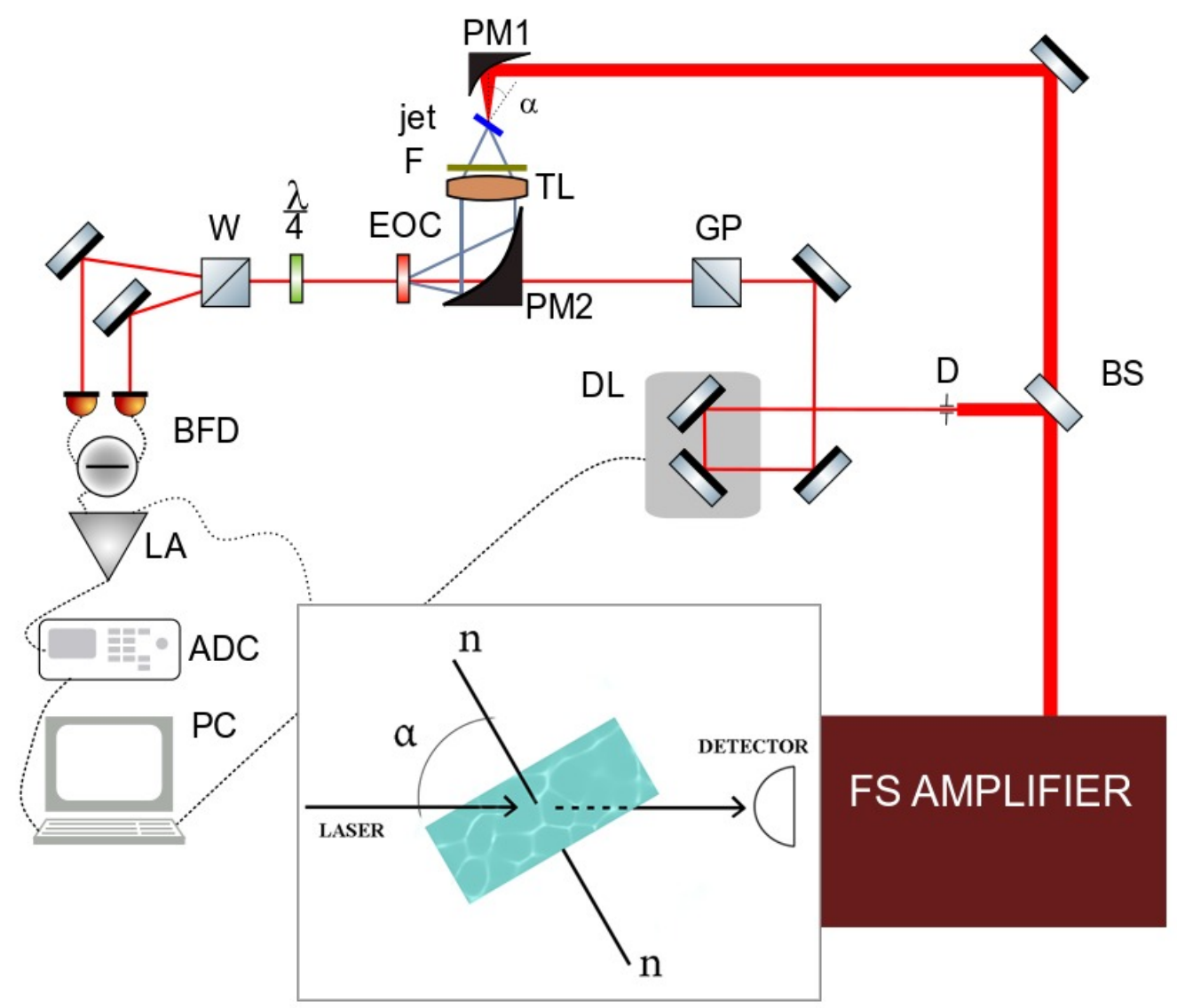

Fig. 1 The scheme of the experimental setup for measuring the generation of terahertz radiation in liquid jets. BS-beam splitter (ratio of energy in the channels 1:99, for probe and pumping, respectively), PM1 - parabolic mirror $\mathrm{f}=5 \mathrm{~cm}, \mathrm{~F}$ - black Teflon filter, TL - TPX lens $\mathrm{f}=5 \mathrm{~cm}, \mathrm{PM} 2$ - parabolic mirror $\mathrm{f}=12 \mathrm{~cm}, \mathrm{EOC}$ - electro-optical crystal ZnTe thickness is $1 \mathrm{~mm}, \mathrm{D}$ - diaphragm, DL- delay line, GP - Glan prism, $\lambda / 4$ - wave plate, $\mathrm{W}$ - Wollaston prism, BFD - balanced detector Nirvana-2007, LA - lock-in amplifier, ADC - analog-todigital converter ,PC- computer. The inset shows the incident optical angle $(\alpha)$

Concentration dependencies. Fig. 2a shows the dependence of the THz generation on the incident optical angle using the $150 \mu \mathrm{m}$ thickness jet of a mixture of ethanol and water. Corresponding measurements were carried out for water with different ethanol concentration, as well as for the pure water. The measurements were performed with a pump energy of 600 $\mu \mathrm{J}$.

These measurements not only confirm the presence of the optimum angle for effective $\mathrm{THz}$ generation, but also reveal its characteristic shift while the ethanol concentration in the mixture changes.

The important parameter to consider while studying oscillating plasma is an ionization energy of the substance, which defines the number of electrons that can be removed from the atom. It is known that ethanol has an ionization energy approximately twice larger than water [7]. 
Given this fact, we expected an increase in the $\mathrm{THz}$ radiation energy with increasing ethanol concentration in the mixture. Fig. 2b demonstrates the linear nature of this increase and confirms our assumptions.

(a)

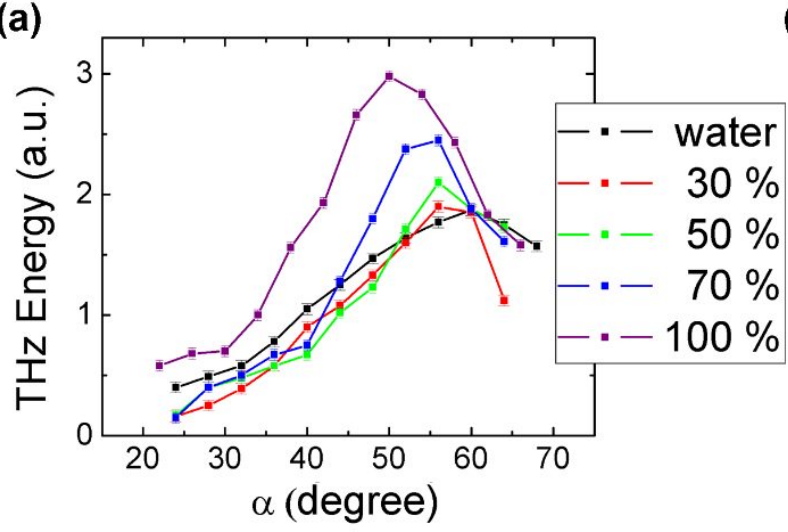

(b)

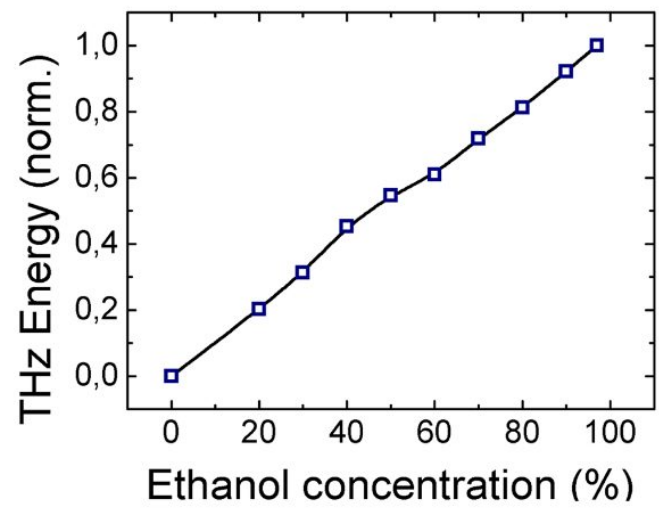

Fig. 2 The study of the effect of ethanol concentration on the output energy of terahertz radiation. a The dependence of the $\mathrm{THz}$ energy in liquid jets of $150 \mu \mathrm{m}$ thickness on the ethanol concentration in mixture. Black line shows the data for pure water. $\mathbf{b}$ The linear $\mathrm{THz}$ energy growth due to the increasing ethanol concentration. Measurements are obtained using pump energy of $600 \mu \mathrm{J}$

In conclusion, we studied the effect of ethanol concentration on the $\mathrm{THz}$ generation efficiency in jets of water and ethanol mixtures. The obtained experimental results clearly show a linear increase in the output energy with an increase in the content of ethanol molecules in water which can be explained by linear replacement of low-efficient charge carriers (water) with highly efficient (ethanol). We believe that this type of dependence occurs due to the presence of a higher ionization energy for ethanol. However, the issues of increasing the efficiency of $\mathrm{THz}$ generation still require careful study, as well as a full consideration of all the necessary optimal conditions for obtaining maximum energies. 


\section{References}

1. Baierl, S. et al. Nonlinear spin control by terahertz-driven anisotropy fields. Nat. Photonics 10, 715-718 (2016).

2. Vicario, C., Ovchinnikov, A. V., Ashitkov, S. I., Agranat, M. B. \& Fortov, V. E. Generation of mJ THz pulses in organic crystal pumped by a Cr:Mg2SiO4 laser. Opt. Lett. 39, 6632-6635 (2014).

3. Kuk, D. et al. Generation of scalable terahertz radiation from cylindrically focused two-color laser pulses in air. Appl. Phys. Lett. 108, 121106 (2016).

4. Qi Jin, Yiwen E, Kaia Williams et al., Applied Physics Letters 111 (7), 071103 (2017).

5. Indranuj Dey, Kamalesh Jana, Vladimir Yu Fedorov et al., Nature Communications 8 (1), 1184 (2017).

6. Yiwen E, Qi Jin, Anton Tcypkin, and X.-C. Zhang Terahertz Wave Generation from Liquid Water Films via Laser-Induced Breakdown, APL2018, in press.

7. Faubel M., Steiner B., and Toennies J. P., The Journal of Chemical Physics 106, 9013 (1997). 